\title{
Tumor Identifier Location
}

National Cancer Institute

\section{Source}

National Cancer Institute. Tumor Identifier Location. NCI Thesaurus. Code C117437.

The site in or on the body at which the tumor identification test or result is performed or collected. 